\title{
Elagolix Treatment for Up to 12 Months in Women With Heavy Menstrual Bleeding and Uterine Leiomyomas
}

James A Simon

The George Washington University

Ayman Al-Hendy

University of Illinois

David F Archer

Eastern Virginia Medical School

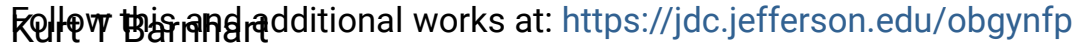

dikersity fhe ennsylvivania

Lief M Brdaleyw how access to this document benefits you

Cleveland Clinic

\section{Recommended Citation}

SireaaxtJange\$dA;alditiendlyaAyiman; Archer, David F; Barnhart, Kurt T; Bradley, Linda D; Carr, Bruce R; Dayspring, Thomas; Feinberg, Eve C; Gillispie, Veronica; Hurtado, Sandra; Kim, JinHee; Liu, Ran; Owens, Charlotte D; Muneyyirci-Delale, Ozgul; Wang, Alice; Watts, Nelson B; and Schlaff, William, "Elagolix Treatment for Up to 12 Months in Women With Heavy Menstrual Bleeding and Uterine Leiomyomas" (2020). Department of Obstetrics and Gynecology Faculty Papers. Paper 80.

https://jdc.jefferson.edu/obgynfp/80

This Article is brought to you for free and open access by the Jefferson Digital Commons. The Jefferson Digital Commons is a service of Thomas Jefferson University's Center for Teaching and Learning (CTL). The Commons is a showcase for Jefferson books and journals, peer-reviewed scholarly publications, unique historical collections from the University archives, and teaching tools. The Jefferson Digital Commons allows researchers and interested readers anywhere in the world to learn about and keep up to date with Jefferson scholarship. This article has been accepted for inclusion in Department of Obstetrics and Gynecology Faculty Papers by an authorized administrator of the Jefferson Digital Commons. For more information, please contact:

JeffersonDigitalCommons@jefferson.edu. 


\section{Authors}

James A Simon, Ayman Al-Hendy, David F Archer, Kurt T Barnhart, Linda D Bradley, Bruce R Carr, Thomas Dayspring, Eve C Feinberg, Veronica Gillispie, Sandra Hurtado, JinHee Kim, Ran Liu, Charlotte D Owens, Ozgul Muneyyirci-Delale, Alice Wang, Nelson B Watts, and William Schlaff 


\title{
Elagolix Treatment for Up to 12 Months in Women With Heavy Menstrual Bleeding and Uterine Leiomyomas
}

\author{
James A. Simon, MD, Ayman Al-Hendy, MD, PhD, David F. Archer, MD, Kurt T. Barnhart, MD, MSCE, \\ Linda D. Bradley, MD, Bruce R. Carr, MD, Thomas Dayspring, MD, Eve C. Feinberg, MD, \\ Veronica Gillispie, MD, Sandra Hurtado, MD, JinHee Kim, MD, Ran Liu, PhD, Charlotte D. Owens, MD, \\ Ozgul Muneyyirci-Delale, MD, Alice Wang, MA, Nelson B. Watts, MD, and William D. Schlaff, MD
}

\begin{abstract}
OBJECTIVE: To investigate the safety and efficacy of elagolix, an oral gonadotropin-releasing hormone antagonist, with hormonal add-back therapy for up to 12 months in women with heavy menstrual bleeding associated with uterine leiomyomas.
\end{abstract}

From the George Washington University, IntimMedicine Specialists, Washington, DC; University of Illinois at Chicago, Chicago, Illinois; Eastern Virginia Medical School, Norfolk, Virginia; Perleman School of Medicine at the University of Pennsylvania, Philadelphia, Pennsylvania; Cleveland Clinic, Cleveland, Ohio; University of Texas Southwestern Medical Center, Dallas, Texas; Attia Medical, San Diego, Califormia; Northweestern University, Chicago, Illinois; Ochsner Health System, New Orleans, Louisiana; University of Texas Health Science Center at Houston, Houston, Texas; Columbia University, New York, New York; AbbVie Inc, North Chicago, Illinois; SUNY Dowenstate Health Science University, Brooklyn, New York; Mercy Health, Cincinnati, Ohio; and Thomas Jefferson University, Philadelphia, Pennsylvania.

AbbVie Inc, funded this study and participated in the study design, research, analysis, data collection, interpretation of data, reviewing, and approval of the publication. All authors had access to relevant data and participated in the writing, review, and approval of this manuscript.

Presented at the 48th American Association of Gynecologic Laparoscopists Global Congress on Minimally Invasive Gynecology, November 10-13, 2019, Vancouver, British Columbia, Canada.

We thank all the women, study investigators, and study team who were involved in this clinical trial. Jeanie K. Meckes, PhD, of AbbVie Inc, provided medical writing assistance for this publication.

Each author has confirmed compliance with the journal's requirements for authorship. Corresponding author: James A. Simon, MD, George Washington University, Washington, DC; email: jsimon@intimmedicine.com.

\section{Financial Disclosure}

James A. Simon has served or is currently serving as a consultant to or on the advisory boards of: AbbVie, Inc, Allergan, Plc, AMAG Pharmaceuticals, Inc, Amgen, Ascend Therapeutics, Bayer HealthCare Pharmaceuticals Inc, CEEK Enterprises, LLC, Covance Inc, Dare' Bioscience, Duchesnay USA, Hologic Inc, KaNDy/NeRRe Therapeutics Ltd, Mitsubishi Tanabe Pharma Development America, Inc, ObsEva SA, Palatin Technologies, Sanofi S.A., Shionogi Inc, Sprout Inc, and TherapeuticsMD. He has also served or is currently serving on the speaker's bureaus of: AbbVie Inc, AMAG Pharmaceuticals, Inc, Duchesnay USA, Novo Nordisk, Shionogi Inc, TherapeuticsMD. He has received or is currently receiving grant/research support from: AbbVie, Inc, Allergan, Plc, Agile Therapeutics, Bayer Healthcare LLC., Endoceutics, Inc, GTX, Inc, Ipsen, Myovant Sciences, New England Research Institute, Inc, ObsEva SA, Palatin Technologies, Symbio Research, Inc, TherapeuticsMD, and Viveve Medical. He is a stockholder in Sermonix Pharmaceuticals. Ayman Al-Hendy has provided consulting services to AbbVie,
METHODS: Elaris UF-EXTEND was a phase 3 extension study that evaluated an additional 6 months (up to 12 months total) of elagolix $300 \mathrm{mg}$ twice daily with hormonal add-back therapy (estradiol $1 \mathrm{mg}$ and norethindrone acetate $0.5 \mathrm{mg}$ once daily) in women who

Allergan, Bayer, Myovant, MD Stem Cells, and he is grant funded by the National Institute of Health for fibroid-related research (R01 ES 028615-01, R01 HD 087417, R01 HD 094378, R01 HD 094380, 5U54 MD 007602-32, R01 HD 100367-01). In addition, he holds a patent for Methods for novel diagnostics and therapeutics for uterine sarcoma (US Pat No. 9,790,562 B2). He was affiliated with Augusta University at the time that the study was completed. David F. Archer has received research support from AbbVie, TherapeuticsMD, Bayer HealthCare, Endoceutics, Glenmark, Shionogi, Symbio and Radius and compensation from AbbVie, TherapeuticsMD, Bayer HealthCare, Endoceutics, Agile Pharmaceuticals, Exeltis/CHEMO France, and TEVA/ HR Pharma for consulting. Kurt T. Barnhart has served as a consultant to AbbVie and Bayer and served on the AbbVie Data and Safety Monitoring Committee. Linda D. Bradley has served as a scientific advisor for AbbVie, Bayer, Allergan, Boston Scientific, Medtronics, Karl Storz, and has received research support from Bayer and royalties from UpToDate, Elsevier, and Wolters Kluwer. Bruce R. Carr has received research support from AbbVie and Syneract, Inc (M360-L102) and served on the Repros Therapeutics Data and Safety Monitoring Board. Thomas Dayspring served as Chief Academic Officer for True Health Diagnostics and a consultant for AbbVie; affiliated with True Health Diagnostics at the time that the study was completed. Eve C. Feinberg has served as a consultant to AbbVie, Natera, and CooperSurgical and served on the AbbVie Data and Safety Monitoring Committee. Veronica Gillispie has served as a study investigator for AbbVie and has served as a consultant to AbbVie. Sandra Hurtado has served as consultant for AbbVie, served on the speaker bureau for AbbVie, Merck, and Bayer, and served as an investigator for AbbVie, Allergan, Amgen, Bayer, Femasys, Ferring, Myovant, Obseva and Therapeutics MD. JinHee Kim has served as a consultant to AbbVie and is a member of the Board of Directors for AAGL. Ran Liu is an AbbVie employee and holds stock or stock options. Charlotte D. Owens is an AbbVie employee and holds stock or stock options. Ozgul Muneyyirci-Delale is a study investigator for AbbVie, Bayer, ObsEva, and Ferring. Alice Wang is an AbbVie employee and holds stock or stock options. Nelson B. Watts has received compensation from Amgen and Radius for speaker honoraria, and from AbbVie, Amgen, Janssen, Radius, and Sanofi for consulting. William D. Schlaff has served as a consultant to AbbVie and has received research support from AbbVie.

(C) 2020 The Author(s). Published by Wolters Kluwer Health, Inc. This is an open-access article distributed under the terms of the Creative Commons Attribution-Non Commercial-No Derivatives License 4.0 (CCBY-NC-ND), where it is permissible to download and share the work provided it is properly cited. The work cannot be changed in any way or used commercially without permission from the journal.

ISSN: 0029-7844/20 
completed an initial 6 months of the same treatment in one of two preceding phase 3 studies. The primary endpoint was the percentage of women with both less than $80 \mathrm{~mL}$ menstrual blood loss during final month and a $50 \%$ or greater reduction in menstrual blood loss from baseline to final month. Safety evaluations included adverse events and bone mineral density changes. The planned sample size of UF-EXTEND was based on estimated rollover and discontinuation rates in the two preceding studies.

RESULTS: From September 2016 to March 2019, 433 women were enrolled in UF-EXTEND. Of these women, 218 received up to 12 months of elagolix with add-back therapy; the mean $\pm \mathrm{SD}$ age of this group was $42.4 \pm 5.4$ years and $67.3 \%$ were black. The percentage of women who met the primary endpoint in this elagolix with addback group was $87.9 \%$ (95\% CI [83.4-92.3]). The most frequently reported adverse events with up to 12 months of elagolix plus add-back therapy were hot flush $(6.9 \%)$, night sweats $(3.2 \%)$, headache $(5.5 \%)$, and nausea $(4.1 \%)$. Mean percent decreases in bone mineral density from baseline to extension month 6 were significantly less with elagolix plus add-back therapy than with elagolix alone \{between-group difference in lumbar spine: -3.3 (95\% $\mathrm{CI}[-4.1$ to -2.5$])\}$.

CONCLUSION: Up to 12 months of elagolix with addback therapy provided sustained reduction in menstrual blood loss in women with uterine leiomyomas, with the addition of add-back therapy attenuating the hypoestrogenic effects of elagolix alone. No new or unexpected safety concerns were associated with an additional 6 months of elagolix with addback therapy.

CLINICAL TRIAL REGISTRATION: ClinicalTrials.gov, NCT02925494.

FUNDING SOURCE: AbbVie Inc funded this study.

(Obstet Gynecol 2020;135:1313-26)

DOI: 10.1097/AOG.0000000000003869

$\bigcup$ terine leiomyomas are the most common noncancerous neoplasms of the uterus affecting up to $80 \%$ of reproductive-age women. ${ }^{1,2}$ They produce a variety of symptoms in about half of these women, the most common being heavy menstrual bleeding, which can lead to anemia. ${ }^{3-5}$ Leiomyomas can have a major effect on a woman's quality of life. ${ }^{6-11}$ Surgery has been the mainstay of leiomyoma treatment, with hysterectomy accounting for almost 70\% of leiomyoma-related procedures. ${ }^{12,13}$ Medical treatment, consisting of oral contraceptives, progestins, tranexamic acid, and gonadotropin-releasing hormone agonists (ie, leuprolide acetate) are often used to provide symptomatic relief; most of which are lim- ited to short-term efficacy. ${ }^{14-16}$ There is a clinical unmet need for an approved long-term oral medical treatment, as an alternative to surgery, that safely and effectively manages heavy menstrual bleeding and improves leiomyoma-related symptoms and quality of life for women with symptomatic leiomyomas.

Elagolix is an oral, nonpeptide gonadotropinreleasing hormone antagonist that results in dosedependent suppression of gonadotropins and ovarian sex hormones and is approved for the management of moderate-to-severe pain related to endometriosis. Here we report results from a phase 3 extension study (Elaris UF-EXTEND) that investigated the efficacy of elagolix $300 \mathrm{mg}$ twice daily with hormonal add-back therapy (estradiol $1 \mathrm{mg}$ and norethindrone acetate $0.5 \mathrm{mg}$ once daily) in reducing heavy menstrual bleeding in women with uterine leiomyomas for up to 12 months of continuous treatment and the longer-term effects of this treatment on hypoestrogenic side effects (bone mineral density decrease and vasomotor symptoms).

\section{ROLE OF THE FUNDING SOURCE}

AbbVie Inc, funded this study and participated in the study design, research, analysis, data collection, interpretation of data, reviewing, and approval of the publication. The authors had access to relevant aggregated study data and other information (such as study protocol, analytic plan and report, validated data table, and clinical study report) required to understand and report research findings. The authors vouch for the presentation and publication of the research findings, have been fully involved at all stages of publication and presentation development, and are willing to take public responsibility for all aspects of the work. All individuals included as authors and contributors who made substantial intellectual contributions to the research, data analysis, and publication or presentation development are listed appropriately. The role of the sponsor in the design, execution, analysis, reporting, and funding is fully disclosed. The authors' personal interests, financial or nonfinancial, relating to this research and its publication have been disclosed.

\section{METHODS}

Elaris UF-EXTEND was conducted at 115 sites in the United States (including Puerto Rico) and Canada from September 2016 to March 2019. This extension study enrolled women who completed 6 months of treatment in one of the two preceding, randomized, double-blind, placebo-controlled, 6-month phase 3 
studies (Elaris UF-1 and UF-2). Women who received elagolix $300 \mathrm{mg}$ twice daily with add-back therapy (estradiol $1 \mathrm{mg}$ and norethindrone acetate $0.5 \mathrm{mg}$ once daily) or elagolix $300 \mathrm{mg}$ twice daily alone in UF-1 or UF-2 continued the same treatment for an additional 6 months in the UF-EXTEND extension study; women on placebo were randomized 1:1 to receive either elagolix with add-back therapy or elagolix alone for up to 6 months of treatment. All women were followed up to 12 months posttreatment. Study participants were provided with a known dose of elagolix; however, the elagolix with add-back dose was blinded. Study site personnel and participants remained blinded to each participant's original treatment from the preceding UF-1 and UF-2 trials and throughout this extension study. This study was approved by the institutional review board or ethics committee for each study site and was conducted in accordance with International Conference on Harmonisation guidelines and applicable regulations and ethical principles of the Declaration of Helsinki.

Here we report results from women who received up to an additional 6 months (12 continuous months total) of elagolix with add-back therapy or elagolix alone in UF-EXTEND; the elagolix alone arm was used to characterize the effect of add-back therapy on hypoestrogenic effects of elagolix. Results from women who received placebo in UF-1 or UF-2 and up to 6 months of elagolix treatment in UF-EXTEND are presented in Appendices 1-4, available online at http://links.lww.com/AOG/B843.

As previously described, up to 6 months of elagolix with add-back therapy achieved statistical significance compared with placebo $(P<.001)$ in reducing menstrual blood loss in women with heavy menstrual bleeding associated with uterine leiomyomas in both UF-1 and UF-2. ${ }^{17}$ In the present manuscript, data from UF-1 and UF-2 were pooled and presented side-by-side with the UF-EXTEND data to assess sustainability of elagolix effects across the 12-month treatment course.

Eligibility criteria for Elaris UF-1 and UF-2 have been previously described. ${ }^{17}$ Briefly, participants were premenopausal women between 18 and 51 years of age who had an ultrasound-confirmed diagnosis of uterine leiomyomas and heavy menstrual bleeding (more than $80 \mathrm{~mL}$ of menstrual blood loss per cycle as measured with alkaline hematin) at the time of screening. Women who completed the 6-month treatment period in UF-1 or UF-2 were eligible to enter UF-EXTEND if they had a bone mineral density decrease less than $8 \%$ in the spine, total hip, and femoral neck, had no clinically significant endometrial pathology on endometrial biopsy, and provided written, informed consent for extended treatment.

All women were instructed to use two forms of nonhormonal contraception (ie, condom with spermicide, diaphragm with spermicide, cervical cap with spermicide) consistently throughout UF-1 and -2 and UF-EXTEND; pregnancy tests were performed monthly at each study visit. In addition, women who were anemic (hemoglobin of less than $12 \mathrm{~g} / \mathrm{dL}$ ) at screening or during the studies were instructed to take iron supplements (300-325 mg of ferrous sulfate); the regimen was determined by the investigator.

All bleeding endpoints were assessed using the alkaline hematin method, which objectively quantifies the amount of blood in used sanitary products collected during the 6-month treatment period of UF-EXTEND and up through the first menses with full menstrual flow in the posttreatment follow-up period. ${ }^{17,18}$ Women who did not return any used sanitary products at any visit during the treatment period or before the first menses in the posttreatment follow-up period were asked a standardized question to determine whether or not they had any menstrual bleeding or spotting since their previous study visit. If no bleeding was indicated over an interval, the menstrual blood loss over the interval was considered as zero.

As in UF-1 and UF-2, the primary efficacy endpoint of UF-EXTEND was the percentage of women who had less than $80 \mathrm{~mL}$ of menstrual blood loss during the final month and a $50 \%$ or greater reduction in menstrual blood loss from baseline to final month. Women who met these two criteria of the primary endpoint but prematurely discontinued study drug because of an adverse event, lack of efficacy, or required surgery or invasive intervention for treatment of uterine leiomyomas were categorized as not having met the primary endpoint. A sensitivity analysis of the primary endpoint was also performed without taking into account reasons for premature discontinuation of the study drug. Secondary efficacy endpoints included change and percent change from baseline in menstrual blood loss to each month and final month, percentage of women with suppression of bleeding (no bleeding, spotting allowed) at the final month, and percentage of women with baseline hemoglobin of $10.5 \mathrm{~g} / \mathrm{dL}$ or less who had an increase in hemoglobin greater than $2 \mathrm{~g} / \mathrm{dL}$ at month 6 . Other efficacy endpoints included the percentage of women with amenorrhea at final month and control of bleeding (no bleeding, up to 1 day of spotting allowed) at final month and change from baseline in the Uterine Fibroid Symptom and Health-Related Quality of Life Questionnaire scores at month 6. ${ }^{17,19}$ 
Average leiomyoma and uterine volume were assessed during UF-EXTEND treatment and posttreatment follow-up periods by pelvic ultrasonography (transabdominal and transvaginal) in all women and by magnetic resonance imaging (MRI) in a subset of women who elected to participate in additional imaging. Average leiomyoma volume was measured based on the average of up to three leiomyomas observed at the given time point. All imaging assessments were read centrally (Parexel International Corporation, Waltham, Massachusetts).

Adverse events were coded using the Medical Dictionary for Regulatory Activities 21.0 and categorized by severity (mild, moderate, or severe) by investigators. Adverse events with new onset (or changed severity) on or after the first dose date and within 30 days after the last dose date were considered treatment-emergent. Serious adverse events were those that were life-threatening, required hospitalization or prolongation of hospitalization, required medical or surgical intervention to prevent serious outcome, or resulted in persistent or significant disability or death. Hot flushes and night sweats were also self-reported at scheduled visits by women in a supplemental questionnaire; women who reported an adverse event of hot flush or night sweats were asked the number and severity of either or both hot flushes and night sweats they experienced over the previous 24 hours at each visit thereafter until resolution of the adverse event.

Bone mineral density in the lumbar spine, total hip, and femoral neck were assessed by dual-energy X-ray absorptiometry scans (GE-Lunar, Madison, Wisconsin; Hologic, Marlborough, Massachusetts), which were performed every 6 months during the treatment and posttreatment follow-up periods of this extension study, and were read centrally (Parexel International Corporation, Waltham, Massachusetts). Clinical laboratory tests, including hemoglobin, lipid panel, and liver function tests, were performed during the screening period of the preceding UF-1 and UF-2 studies, at baseline and each treatment month (or at premature discontinuation), and at applicable posttreatment follow-up visits, and were read centrally (Q2 Solutions, Valencia, California). Endometrial biopsies were performed during screening and at month 6 (or at premature discontinuation) and read centrally (Q2 Solutions).

Approximately 400 women were expected to enroll in the Elaris UF-EXTEND study based on estimated rollover and discontinuation rates in Elaris UF-1 and UF-2. All efficacy and safety analyses included all women who received at least one dose of the study drug in this extension study, including those who prematurely discontinued the study drug or withdrew consent. Baseline values for all measures were defined as before dosing in either UF-1 and UF-2 for women who received elagolix treatment in those studies (for women who received placebo in UF-1 or UF-2, baseline was defined as the last value before the first dose of elagolix in UF-EXTEND, unless otherwise specified). Final month was defined as the last 28 days before and including the last dose date in UFEXTEND.

Statistical comparisons between the elagolix with add-back therapy and elagolix-alone groups that received up to 12 months of treatment were not prespecified and therefore were not performed in UFEXTEND, as the study was not designed for these analyses, except when evaluating changes in bone mineral density. The primary endpoint and secondary endpoints of categorical assessments (eg, percentage of women with suppression of bleeding at the final month and percentage of women with baseline hemoglobin of $10.5 \mathrm{~g} / \mathrm{dL}$ or less who had an increase in hemoglobin greater than $2 \mathrm{~g} / \mathrm{dL}$ at month 6 ) were summarized by frequency and percentages. The secondary endpoints of all change and percent change from baseline analyses were summarized with descriptive statistics. Statistical testing was only performed for percent changes in bone mineral density using SAS 9.4 with an analysis of covariance model with treatment as the main effect and baseline value as a covariate with a two-sided significance level of 0.05 and a $95 \%$ CI.

\section{RESULTS}

A total of 433 women who completed the 6-month treatment period in Elaris UF-1 or UF-2 were enrolled and treated in Elaris UF-EXTEND (Fig. 1). Of these women, 218 were treated with elagolix plus add-back therapy (98 with elagolix alone) in the preceding studies and continued the same treatment for up to an additional 6 months in UF-EXTEND; thus, were included in this analysis. Similar proportions of women in each treatment group $(16.5 \%$ for elagolix with add-back therapy and $19.4 \%$ for elagolix alone) discontinued the study drug, with the most common primary reason for discontinuation being lost to follow-up (5.0\% and 5.1\% respectively). As summarized in Table 1, the demographics and baseline characteristics of women enrolled in UF-EXTEND were generally well-balanced across the treatment groups and similar to those of the overall population of UF1 and UF-2. 


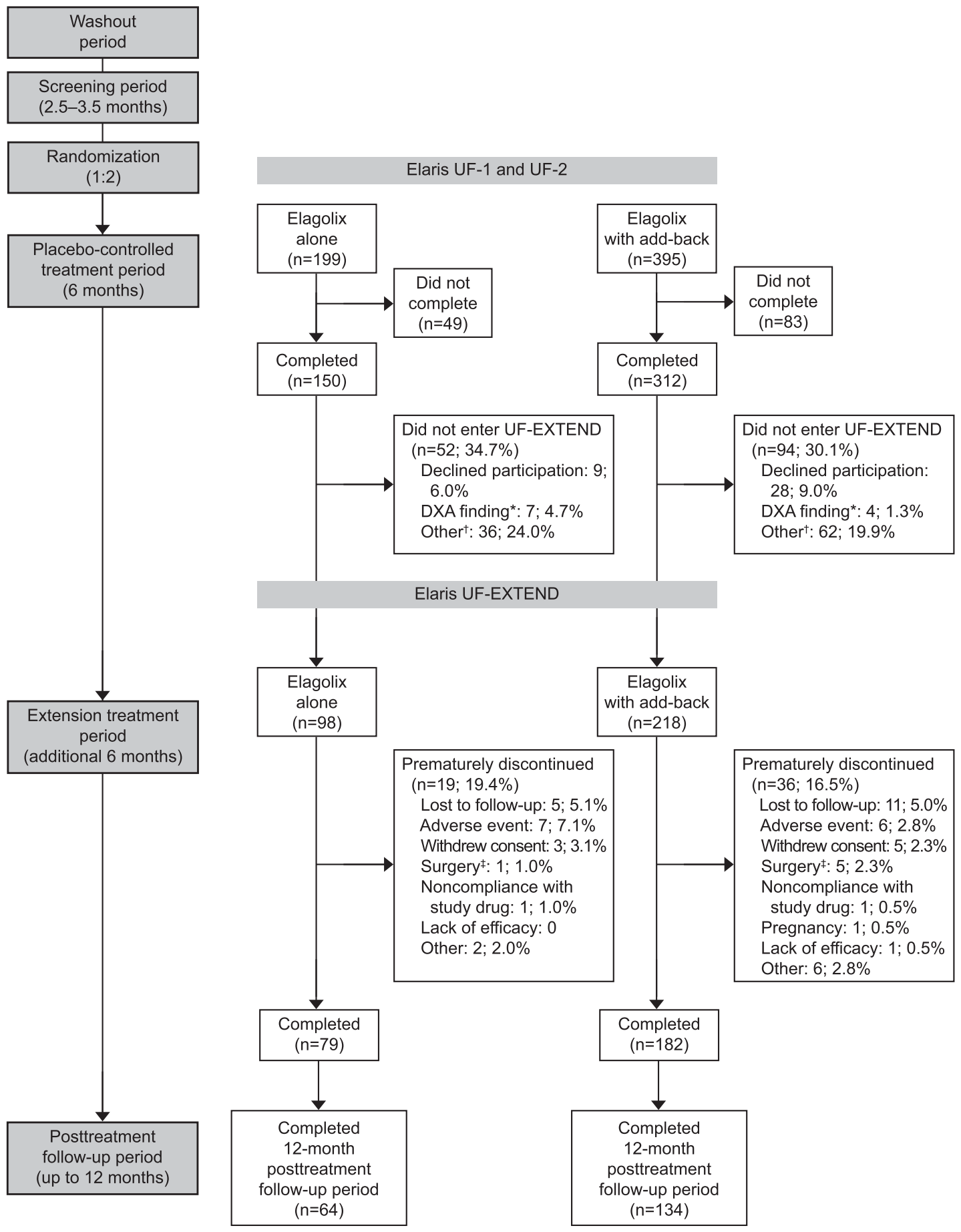

Fig. 1. Study design and disposition of women treated with up to 12 months of elagolix. Elagolix alone, elagolix $300 \mathrm{mg}$ twice daily throughout UF-1 and UF-2 and UF-EXTEND; elagolix with add-back therapy, elagolix $300 \mathrm{mg}$ twice daily with add-back therapy (estradiol $1 \mathrm{mg}$ and norethindrone $0.5 \mathrm{mg}$ once daily) throughout UF-1 and UF-2 and UF-EXTEND. *In UF-1 and UF-2 combined, 5 of $150(3.3 \%)$ women treated with elagolix alone and 3 of $312(1.0 \%)$ women treated with elagolix plus add-back therapy did not enter UF-EXTEND because of a dual-energy X-ray absorptiometry scan (DXA) finding of bone mineral density decrease greater than $8 \%$ in at least one of the anatomic locations (lumbar spine, total hip, and femoral neck). ${ }^{+}$The most common other reason was that the extension study was closed to enrollment. ${ }^{*}$ Women required surgery or invasive intervention for treatment of uterine leiomyomas. Other reasons for premature discontinuation included moved out of state $(n=2)$, weight exceeded DXA limits, study site closing, participant stopped drug because she thought she was pregnant but was not, did not like bleeding while on medication, personal reasons, and DXA results.

Simon. Twelve Months of Elagolix With Add-Back Therapy. Obstet Gynecol 2020. 
Table 1. Patient Demographics and Baseline Characteristics in Women Treated With Up to 12 Months of Elagolix*

\begin{tabular}{|c|c|c|}
\hline & Elagolix Alone $(n=98)$ & Elagolix With Add-Back Therapy $(n=218)$ \\
\hline Age (y) & $42.2 \pm 5.4$ & $42.4 \pm 5.4$ \\
\hline \multicolumn{3}{|l|}{ Race } \\
\hline White & $25(25.8)$ & $65(30.0)$ \\
\hline Black or African American & $70(72.2)$ & $146(67.3)$ \\
\hline Other & $2(2.0)$ & $6(2.8)$ \\
\hline Missing & 1 & 1 \\
\hline BMI $\left(\mathrm{kg} / \mathrm{m}^{2}\right)$ & $35.4 \pm 7.5$ & $33.7 \pm 6.8(n=217)$ \\
\hline $\mathrm{MBL}(\mathrm{mL})$ & $260.9 \pm 181.5$ & $224.7 \pm 147.9$ \\
\hline Hemoglobin $(\mathrm{g} / \mathrm{dL})$ & $10.8 \pm 1.6$ & $11.1 \pm 1.5$ \\
\hline \multicolumn{3}{|l|}{ Uterine volume $\left(\mathrm{cm}^{3}\right)$} \\
\hline TAU, TVU & $594.4 \pm 589.3$ & $485.1 \pm 381.2$ \\
\hline MRI & $830.5 \pm 980.9(n=52)$ & $590.5 \pm 441.5(n=110)$ \\
\hline \multicolumn{3}{|l|}{ Average leiomyoma volume $\left(\mathrm{cm}^{3}\right)$} \\
\hline TAU/TVU & $68.7 \pm 137.5(n=97)$ & $54.5 \pm 72.5(n=213)$ \\
\hline MRI & $91.2 \pm 127.3(n=49)$ & $73.6 \pm 64.3(n=99)$ \\
\hline \multicolumn{3}{|l|}{ UFS-QOL score } \\
\hline Symptom severity & $63.6 \pm 20.9(n=97)$ & $58.4 \pm 21.6(n=217)$ \\
\hline Total HRQOL & $41.5 \pm 24.2(n=97)$ & $44.4 \pm 24.0(n=217)$ \\
\hline \multicolumn{3}{|l|}{ BMD Z-score } \\
\hline Lumbar spine & $1.0 \pm 1.1$ & $1.1 \pm 1.1$ \\
\hline Total hip & $0.9 \pm 1.0$ & $0.8 \pm 0.9$ \\
\hline Femoral neck & $0.7 \pm 0.9$ & $0.7 \pm 0.9$ \\
\hline
\end{tabular}

BMI, body mass index; MBL, menstrual blood loss; TAU, transabdominal ultrasonography; TVU, transvaginal ultrasonography; MRI, magnetic resonance imaging; UFS-QOL, Uterine Fibroid Symptom and Quality of Life Questionnaire; HRQOL, health-related quality of life; $B M D$, bone mineral density.

Data are mean \pm SD or $\mathrm{n}(\%)$.

Demographics and baseline characteristics for all women are from before first dosing in Elaris UF-1 or UF-2. For uterine volume, average leiomyoma volume, and UFS-QoL scores, if no measurement was collected on or before day 1 in UF-1 or -2 , the first measurement collected before day 8 in UF-1 or -2 was used as baseline.

*Elagolix alone=elagolix $300 \mathrm{mg}$ twice daily throughout UF-1 and -2 and UF-EXTEND; elagolix with add-back therapy=elagolix $300 \mathrm{mg}$ twice daily with add-back (estradiol $1 \mathrm{mg}$ and norethindrone $0.5 \mathrm{mg}$ once daily) throughout UF-1 and -2 and UF-EXTEND.

Of the women who received up to 12 months of criteria of the primary endpoint was $87.9 \%$ (95\% CI elagolix with add-back therapy in Elaris UFEXTEND, the proportion who met the two bleeding [83.4-92.3], $\mathrm{n}=206)$ and $89.4 \%$ (95\% CI [83.1-95.6], $\mathrm{n}=94$ ) for elagolix alone; Appendix 5, available

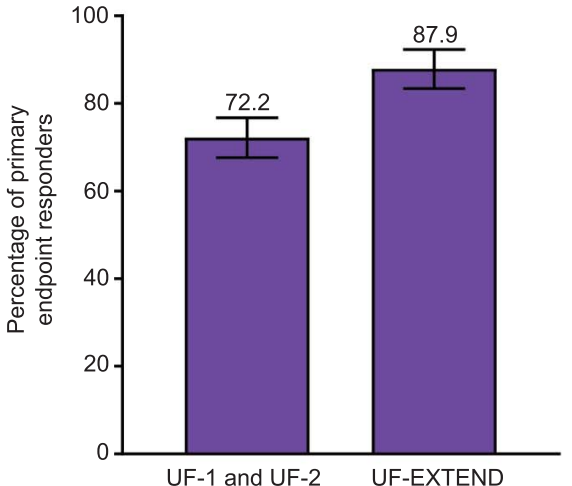

A

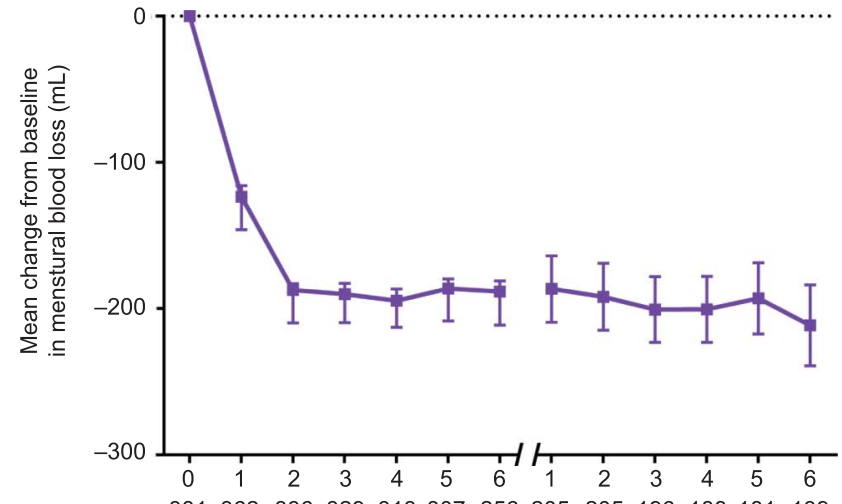

B

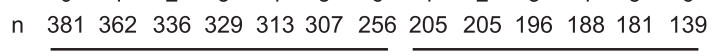

Fig. 2. Percentage of women who met the primary endpoint $(\mathbf{A})$ and mean change from baseline in menstrual blood loss in women treated with up to 12 months of elagolix with add-back therapy (B). Data are \% or mean with error bars indicating $95 \% \mathrm{Cl}$. Baseline was before first dosing in the UF-1 and UF-2 studies. *Mean changes from baseline in menstrual blood loss for UF-1 and UF-2 are presented as least squares means.

Simon. Twelve Months of Elagolix With Add-Back Therapy. Obstet Gynecol 2020. 
online at http://links.lww.com/AOG/B843); these results were consistent with the results pooled from UF1 and UF-2 $272.2 \%$ (95\% CI [67.7-76.7]) for elagolix with add-back therapy and $80.6 \%(95 \%$ CI [74.9-86. 2]) for elagolix alone\} (Fig. 2A; Appendix 5 [http:// links.lww.com/AOG/B843]). The results of a sensitivity analysis of the primary endpoint which did not consider reasons for premature discontinuation of the study drug were similar to those of the primary analysis (Appendix 6, available online at http://links. lww.com/AOG/B843). Results of the primary endpoint from women who received placebo in either UF-1 or UF-2 and up to 6 months of elagolix treatment in UF-EXTEND are presented in Appendix 3 (http://links.lww.com/AOG/B843).

Mean reductions in menstrual blood loss seen at month 6 of UF-1 and UF-2 were sustained through an additional 6 months of elagolix with add-back therapy during UF-EXTEND; mean changes from baseline ranged from $-186.5 \mathrm{~mL}(95 \% \mathrm{CI}[-209.2$ to -163.9$])$ at extension month 1 to $-211.4 \mathrm{~mL}(95 \%$ CI [ -239.1 to -183.7$]$ ) at extension month 6 (Fig. 2B; mean percent change in Appendix 7, available online at http:// links.lww.com/AOG/B843). The majority of women receiving elagolix with add-back therapy achieved suppression of bleeding $\{74.8 \%$ (95\% CI [68.8-80. 7])\} at the final month of UF-EXTEND; the corresponding percentage at the final month of UF-1 and UF-2 combined was $58.9 \%$ (95\% CI [53.8-64.0]) (Table 2). Additionally, the majority of women receiving elagolix with add-back therapy who had a low baseline hemoglobin level of $10.5 \mathrm{~g} / \mathrm{dL}$ or less showed an increase from baseline greater than $2 \mathrm{~g} / \mathrm{dL}$ at UFEXTEND month $6(72.5 \%, 95 \%$ CI [60.3-84.8]); the corresponding percentage at month 6 of UF-1 and UF-2 combined was 56.0\% (95\% CI [46.3-65.7]) (Table 2). Results of secondary and other efficacy endpoints from women who received elagolix alone up to 12 months are presented in Appendix 5 (http://links. lww.com/AOG/B843) and in Appendix 3 (http:// links.lww.com/AOG/B843) for women who received placebo in UF-1 or UF-2 and up to 6 months of elagolix treatment in UF-EXTEND.

More than half of the women who received up to 12 months of elagolix with add-back therapy achieved amenorrhea $(64.6 \%, 95 \%$ CI [58.0-71.1]) and control of bleeding $(64.6 \%, 95 \% \mathrm{CI}[58.0-71.1])$ at the final month of UF-EXTEND, respectively. In the elagolix with add-back group, there was a small increase in mean average leiomyoma volume from baseline to UF-EXTEND month $6\left(1.5 \mathrm{~cm}^{3}\right.$ [95\% CI -6.0 to 8.9] measured by ultrasound scan and $9.4 \mathrm{~cm}^{3}[95 \%$ CI -1.2 to 20.0 ] by MRI); however, mean uterine volume was reduced at UF-EXTEND month 6 $\left\{-57.3 \mathrm{~cm}^{3}(95 \% \mathrm{CI}[-85.0\right.$ to -29.5$])$ by ultrasound scan and $-30.0 \mathrm{~cm}^{3}$ (95\% CI [-65.8 to 5.8$]$ ) by MRI (Appendix 8, available online at http://links.lww. com/AOG/B843). Elagolix with add-back treatment was also associated with improved quality of life based on mean changes from baseline to UF-EXTEND month 6 in Uterine Fibroid Symptom and HealthRelated Quality of Life Questionnaire scores, including symptom severity score and total HRQL score (subscales included Concern, Activities, Energy and

Table 2. Secondary and Other Efficacy Endpoints After 6 Months and 12 Months of Elagolix Treatment in Women Treated With Up to 12 months of Elagolix*

Endpoint (Timepoint)

Elagolix With Add-Back Therapy

Women with amenorrhea

UF-1 and -2 final month

UF-EXTEND final month

$50.4(45.2-55.6)$

Women with control of bleeding

UF-1 and -2 final month

UF-EXTEND final month

$64.6(58.0-71.1)$

Women with suppression of bleeding

UF-1 and -2 final month

$(45.2-55.6)$

$64.6(58.0-71.1)$

UF-EXTEND final month

$58.9(53.8-64.0)$

$74.8(68.8-80.7)$

Women with baseline hemoglobin of $10.5 \mathrm{~g} / \mathrm{dL}$ or less who had an increase of more than $2 \mathrm{~g} / \mathrm{dL}^{+}$

UF-1 and -2 month 6

$56.0(46.3-65.7)$

UF-EXTEND month 6

$72.5(60.3-84.8)$

Data are \% $(95 \% \mathrm{Cl})$.

*Elagolix with add-back therapy=elagolix $300 \mathrm{mg}$ twice daily with add-back (estradiol $1 \mathrm{mg}$ and norethindrone $0.5 \mathrm{mg}$ once daily) throughout UF- 1 and -2 and UF-EXTEND.

${ }^{+}$Baseline was before first dosing in UF-1 and UF-2 studies. 
mood, Control, Self-conscious, Sexual function) (Appendix 9, available online at http://links.lww. com/AOG/B843).

Furthermore, of the women who received up to an additional 6 months of elagolix with add-back therapy during UF-EXTEND and entered the posttreatment follow-up period $(\mathrm{n}=184)$, the majority of these women $(79.3 \%)$ reported a first posttreatment menses within 2 months after stopping the study drug; mean volume of the first posttreatment menses was $198.7 \mathrm{~mL}$.

Approximately $60 \%$ of women who received up to 12 months of elagolix with add-back therapy or elagolix alone had at least one treatment-emergent adverse event with an onset on or after the first dose of study drug in this extension study (Table 3). The most frequently reported adverse events with elagolix plus add-back therapy were hot flush and headache; which are consistent with those of UF-1 and UF-2. The most common adverse events leading to treatment discontinuation during the second 6 months of treatment were bone density decrease (elagolix alone: four [4.1\%]; elagolix with add-back therapy: one [0.5\%]) and depression (elagolix alone: one [1.0\%]; elagolix with add-back therapy: one $[0.5 \%])$. The majority of adverse events were considered nonserious and mild or moderate in severity by investigators. Serious adverse events that led to study drug discontinuation included breast cancer $(n=1)$, menorrhagia and pelvic pain $(\mathrm{n}=1)$, and hysterectomy $(\mathrm{n}=1)$; all of which occurred in the elagolix with add-back group. The profile of adverse events from women who received placebo in UF-1 or UF-2 and up to 6 months of elagolix treatment in UF-EXTEND are presented in Appendix 4 (http://links.lww.com/AOG/B843). The rate of adverse events over the course of 12 months of elagolix treatment are listed in Appendix 10, available online at http://links.lww.com/AOG/B843.

There was one pregnancy that was reported during the treatment period of UF-EXTEND; a woman had a positive urine pregnancy test confirmed by a positive serum pregnancy test on day 90 , discontinued the study drug the same day, and had a spontaneous abortion on day 95 (Posttreatment day 6). No deaths were reported in the elagolix treatment groups during the extension study.

Among women who received up to 12 months of elagolix treatment, the overall rate of either or both hot flushes and night sweats with new onset in this extension study were reported by $7.8 \%$ of women in the elagolix with add-back group and $5.1 \%$ in the elagolix-alone group; none of these women discontinued the study drug because of either or both hot flush and night sweat events, as none of the events were considered serious by investigators. Based on a supplemental form completed by the women themselves, fewer women in the elagolix with addback group (range, $n=19-29$ ) reported either or both hot flush and night sweats every month during the treatment period even though the elagolix with addback group had approximately twice as many women as the elagolix-alone group (range, $\mathrm{n}=37-49$, Appendix 11, available online at http://links.lww. com/AOG/B843). Participant-reported supplemental form data also showed that a smaller percentage of women in the elagolix with add-back group than in the elagolix-alone group had a maximal severity of moderate or severe for either or both hot flush and night sweat events at most treatment months. The mean number of these events were lower in the

Table 3. Overview of Treatment-Emergent Adverse Events During UF-EXTEND for Women Who Received Up to 12 Months of Elagolix*

\begin{tabular}{lcc} 
Adverse Event & $\begin{array}{c}\text { Elagolix Alone } \\
(\mathbf{n = 9 8})\end{array}$ & $\begin{array}{c}\text { Elagolix With Add-Back Therapy } \\
(\mathbf{n = 2 1 8})\end{array}$ \\
\hline Any & $60(61)$ & $134(61)$ \\
$\quad$ Any serious & $2(2)$ & $6(3)$ \\
Any severe & $3(3)$ & $14(6)$ \\
Any leading to study drug discontinuation & $7(7)$ & $8(4)$ \\
$\quad$ Any fatal & 0 & 0 \\
Occurred in 5\% or more of participants across elagolix treatment & & $15(7)$ \\
$\quad$ groups & $3(3)$ & $12(6)$ \\
$\quad$ Hot flush & $4(4)$ & \\
\hline
\end{tabular}

Data are $\mathrm{n}(\%)$.

Adverse events are listed by patient incidence in descending order by elagolix with add-back therapy. Only treatment-emergent adverse events that occurred (or changed severity) during UF-EXTEND are presented here.

* Elagolix alone=elagolix $300 \mathrm{mg}$ twice daily throughout UF-1 and -2 and UF-EXTEND; elagolix with add-back therapy=elagolix 300 mg twice daily with add-back (estradiol $1 \mathrm{mg}$ and norethindrone $0.5 \mathrm{mg}$ once daily) throughout UF- 1 and -2 and UF-EXTEND. 
elagolix with add-back group than in the elagolixalone group at most months (Appendix 12, available online at http://links.lww.com/AOG/B843).

The lumbar spine mean bone mineral density percent change from baseline for the elagolix alone treatment group was $-4.8 \%(95 \%$ CI [ -5.4 to -4.1$]$; median Z-score 0.5$)$ and for elagolix with add-back therapy $-1.5 \%(95 \%$ CI $[-1.9$ to -1.0$]$; median Zscore 0.8 ) in women who received up to 12 months of treatment at UF-EXTEND month 6 (Fig. 3). At the same anatomical location, at posttreatment month 6 the mean bone mineral density percent change from baseline for the elagolix alone treatment group was $-2.8 \%(95 \%$ CI [ -3.6 to -1.9$]$; median Z-score 0.5$)$ and for elagolix with add-back therapy $-0.6 \%(95 \%$ CI $[-1.1$ to -0.1$]$; median Z-score 1.0$)$, and at posttreatment Month 12, $-2.0 \%(95 \%$ CI [ -2.8 to -1.2$]$; median Z-score 0.6$)$, and $-0.6 \%(95 \%$ CI [ -1.1 to $-0.0]$; median Z-score 1.1), respectively. Similar trends were seen at the total hip and femoral neck locations (Figs. 4 and 5).

Bone mineral density changes from baseline were less with elagolix with add-back therapy compared with elagolix alone; the differences between groups were statistically significant. For all locations, a higher percentage of women in the elagolix with add-back group than in the elagolix-alone group had an increase or no change in bone mineral density from baseline to UF-EXTEND month 6. A smaller percentage of women in the elagolix with add-back group than in the elagolix-alone group had more than 3\% bone mineral density decreases at UF-EXTEND month 6 (Appendix 13, available online at http:// links.lww.com/AOG/B843). Thirty women treated with elagolix for up to 12 months (23 in the elagolix-alone group and seven in the elagolix with add-back group) had an $8 \%$ or greater bone mineral density decrease at one or more anatomic locations during the 6 months of UF-EXTEND, of whom five (four in the elagolix-alone group and one in the elagolix with add-back group) had a Z-score of -1.5 or less in the same location where they had the $8 \%$ or greater bone mineral density decrease. None of these 30 women had bone fractures. At UF-EXTEND posttreatment months 6 and 12 for all anatomical locations, no women at any anatomical location had a Zscore less than -2.0 , the lower bound of the normal age- and race-matched range (Figs. $3-5) .{ }^{20}$

Women who received up to 12 months of elagolix treatment had transient elevations in low-density lipoprotein cholesterol (LDL-C) and apolipoprotein $\mathrm{B}$ that generally occurred within the first 3 months of treatment during UF-1 and UF-2 and then stabilized.
The mean increases in these serum lipid levels were lower with elagolix plus add-back therapy than with elagolix alone (Appendix 14, available online at http://links.lww.com/AOG/B843). In the elagolix with add-back group, both mean LDL-C and apolipoprotein $\mathrm{B}$ levels returned to near baseline levels by posttreatment follow-up month 3. Mean triglyceride levels were generally similar to baseline in the elagolix with add-back group and were lower than in the elagolix-alone group.

None of the women discontinued the study drug because of an adverse event of anemia. In women receiving up to 12 months of elagolix, treatment led to increases in mean hemoglobin levels from baseline (Appendix 15, available online at http://links.lww. com/AOG/B843).

Two women who received up to 12 months of elagolix treatment (one in the elagolix with add-back group and one in the elagolix-alone group) had elevations in liver transaminase levels during UFEXTEND that were more than three times or more than five times the upper limit normal; neither had concurrent elevations in bilirubin and no pattern in time to onset was identified. In both of these women, their elevations were asymptomatic and declined within 1-4 months after peak values, regardless of study drug continuation. Of these two women, only the one in the elagolix-alone group discontinued the study drug because of liver transaminase elevations; her levels returned to normal after the study drug was discontinued. Further, there were no cases of severe liver injury (ie, irreversible liver failure that is fatal or requires liver transplantation) reported in UFEXTEND, and no one met the biochemical criteria for Hy's law.

Results from the endometrial biopsy showed no evidence of hyperplasia or malignancy at baseline or UF-EXTEND month 6 and no treatment-emergent adverse event of ovarian cysts were reported during the extension study. Despite cycle-related differences, mean endometrial thickness, as measured by ultrasound scan or MRI, decreased from baseline to UF-EXTEND month 6 in each of the two elagolix groups that received up to 12 months of treatment (Appendix 16, available online at http://links.lww.com/AOG/B843).

\section{DISCUSSION}

Previous reports of two phase 3 studies (Elaris UF-1 and UF-2) showed that up to 6 months of elagolix 300 $\mathrm{mg}$ twice daily with hormonal add-back therapy $(1 \mathrm{mg}$ estradiol and $0.5 \mathrm{mg}$ norethindrone acetate once daily), when compared with placebo, significantly reduced menstrual bleeding and improved quality of 

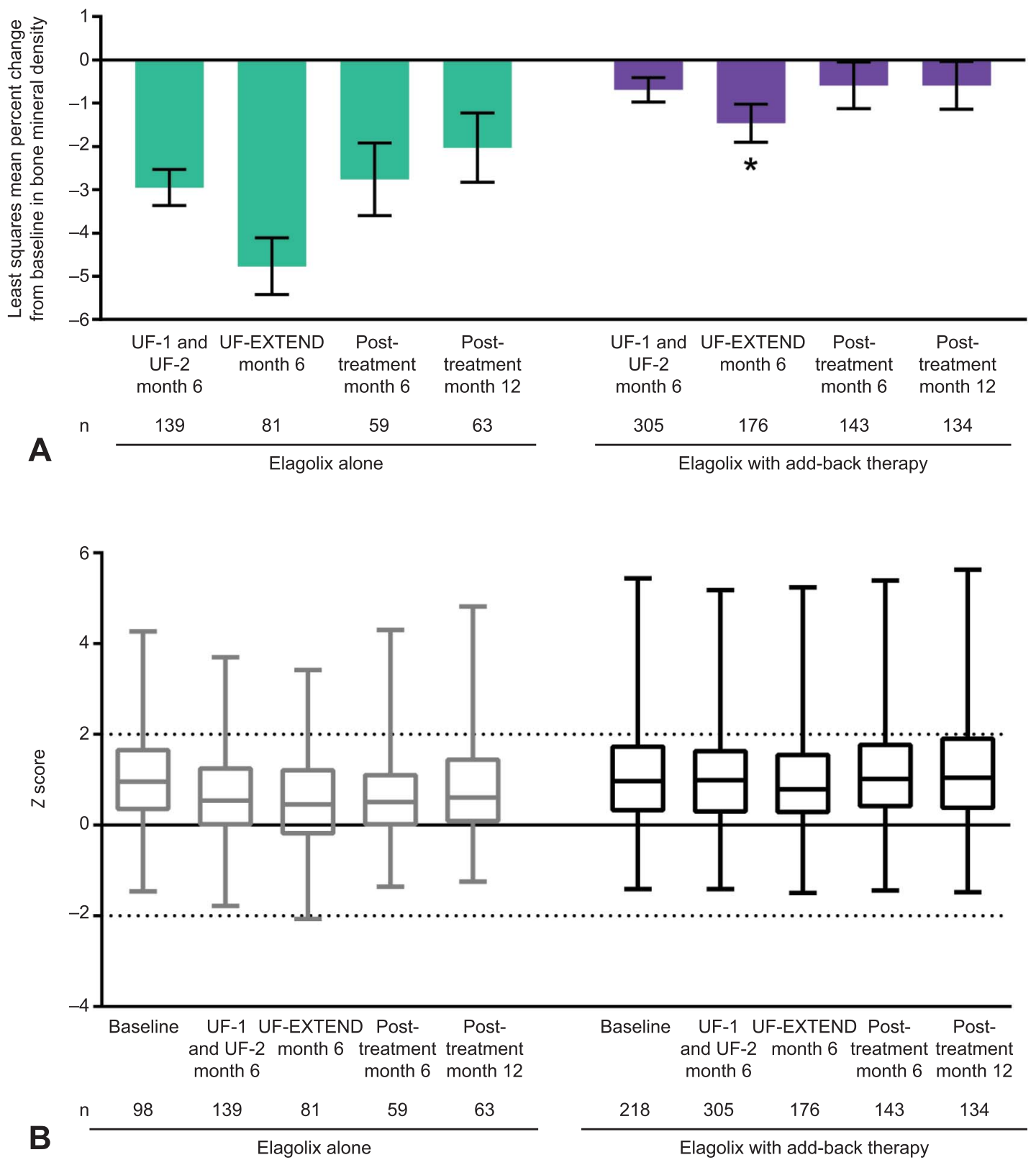

Fig. 3. Lumbar spine bone mineral density changes from baseline $(\mathbf{A})$ and bone mineral density Z-scores during treatment and posttreatment periods in women treated with up to 12 months of elagolix (B). Bone mineral density changes are presented as least square mean percent change, with error bars indicating $95 \% \mathrm{Cl}$. Bone mineral density Z-scores are presented as median, quartile 1, quartile 3, and range. Bone mineral density changes and Z-scores for UF-1 and UF-2 exclude participants who switched machine types. Elagolix alone, elagolix $300 \mathrm{mg}$ twice daily throughout UF-1 and UF-2 and UF-EXTEND; elagolix with add-back therapy, elagolix $300 \mathrm{mg}$ twice daily with add-back therapy (estradiol $1 \mathrm{mg}$ and norethindrone $0.5 \mathrm{mg}$ once daily) throughout UF-1 and UF-2 and UF-EXTEND. Baseline was before first dosing in UF-1 and UF-2 studies. ${ }^{*} P \leq .05$ for test of difference between the elagolix with add-back therapy and elagolix-alone groups using an analysis of covariance model with treatment as the main effect and baseline value as a covariate for extension month 6 .

Simon. Twelve Months of Elagolix With Add-Back Therapy. Obstet Gynecol 2020.

life in women with heavy menstrual bleeding associated with uterine leiomyomas. Herein, this corresponding extension study demonstrated maintenance of these effects for an additional 6 months (up to 12 months total) of elagolix with add-back treatment. In women who received up to 12 months of elagolix with add-back therapy, the majority of women $(87.9 \%)$ met the primary endpoint of having both less than $80 \mathrm{~mL}$ 

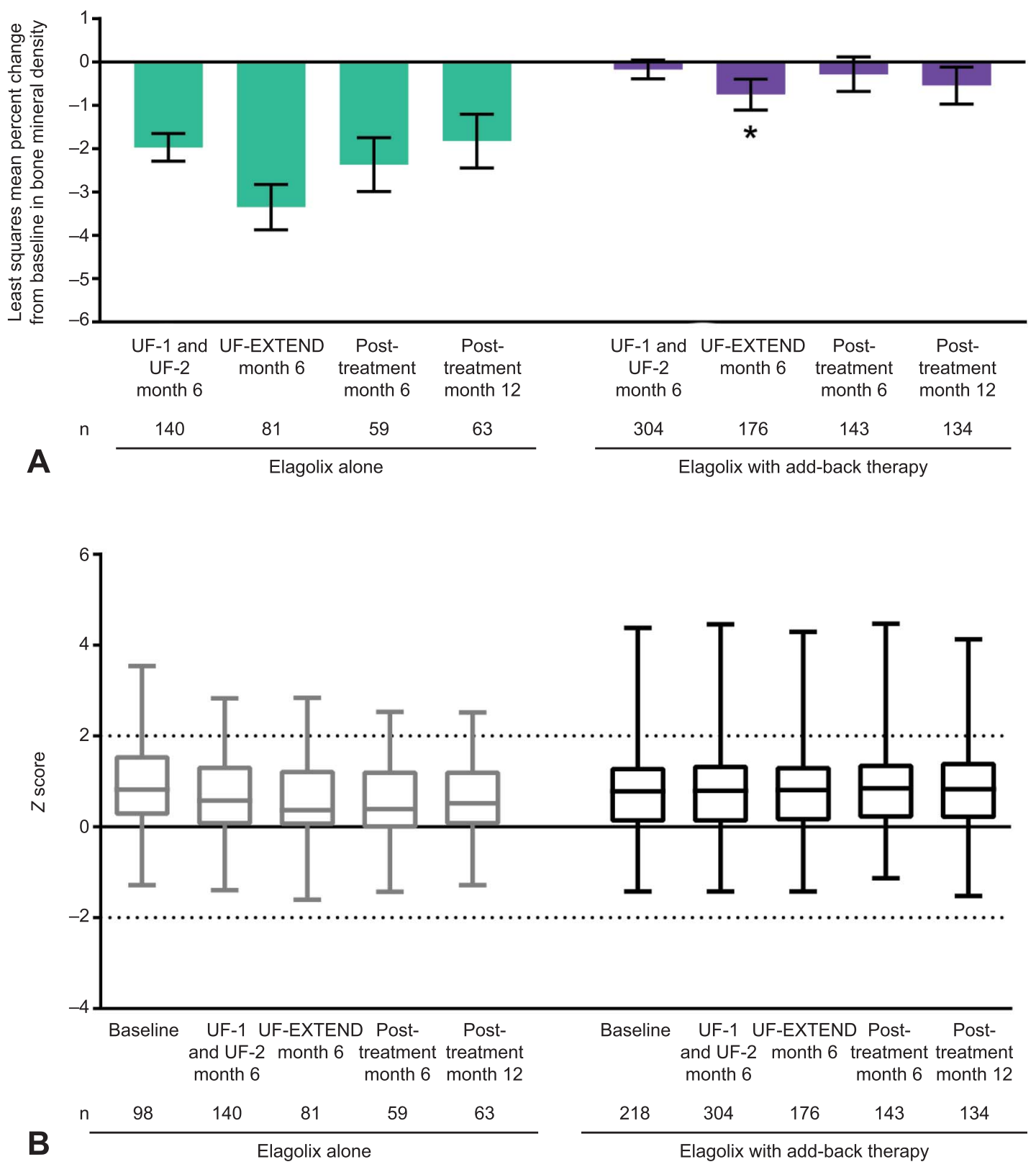

Fig. 4. Total hip bone mineral density changes from baseline $(\mathbf{A})$ and bone mineral density Z-scores during treatment and posttreatment periods in women treated with up to 12 months of elagolix (B). Bone mineral density changes are presented as least square mean percent change with error bars indicating $95 \% \mathrm{Cl}$. Bone mineral density Z-scores are presented as median, quartile 1, quartile 3, and range. Bone mineral density changes and Z-scores for UF-1 and UF-2 exclude participants who switched machine types. Elagolix alone, elagolix $300 \mathrm{mg}$ twice daily throughout UF-1 and UF-2 and UF-EXTEND; elagolix with add-back therapy, elagolix $300 \mathrm{mg}$ twice daily with add-back therapy (estradiol 1 mg and norethindrone $0.5 \mathrm{mg}$ once daily) throughout UF-1 and UF-2 and UF-EXTEND. Baseline was before first dosing in UF-1 and UF-2 studies. ${ }^{*} P \leq .05$ for test of difference between the elagolix with add-back therapy and elagolix-alone groups using an analysis of covariance model with treatment as the main effect and baseline value as a covariate for extension month 6 . Simon. Twelve Months of Elagolix With Add-Back Therapy. Obstet Gynecol 2020.

of menstrual blood loss at the final month and a 50\% or greater reduction in menstrual blood loss from baseline to final month. Reductions in menstrual blood loss were consistent across the 12-month treatment course. Similar to the results of the preceding UF-1 and UF-2 studies, most women treated with elagolix with add-back therapy achieved suppression of bleeding $(74.8 \%)$ at the final month in 

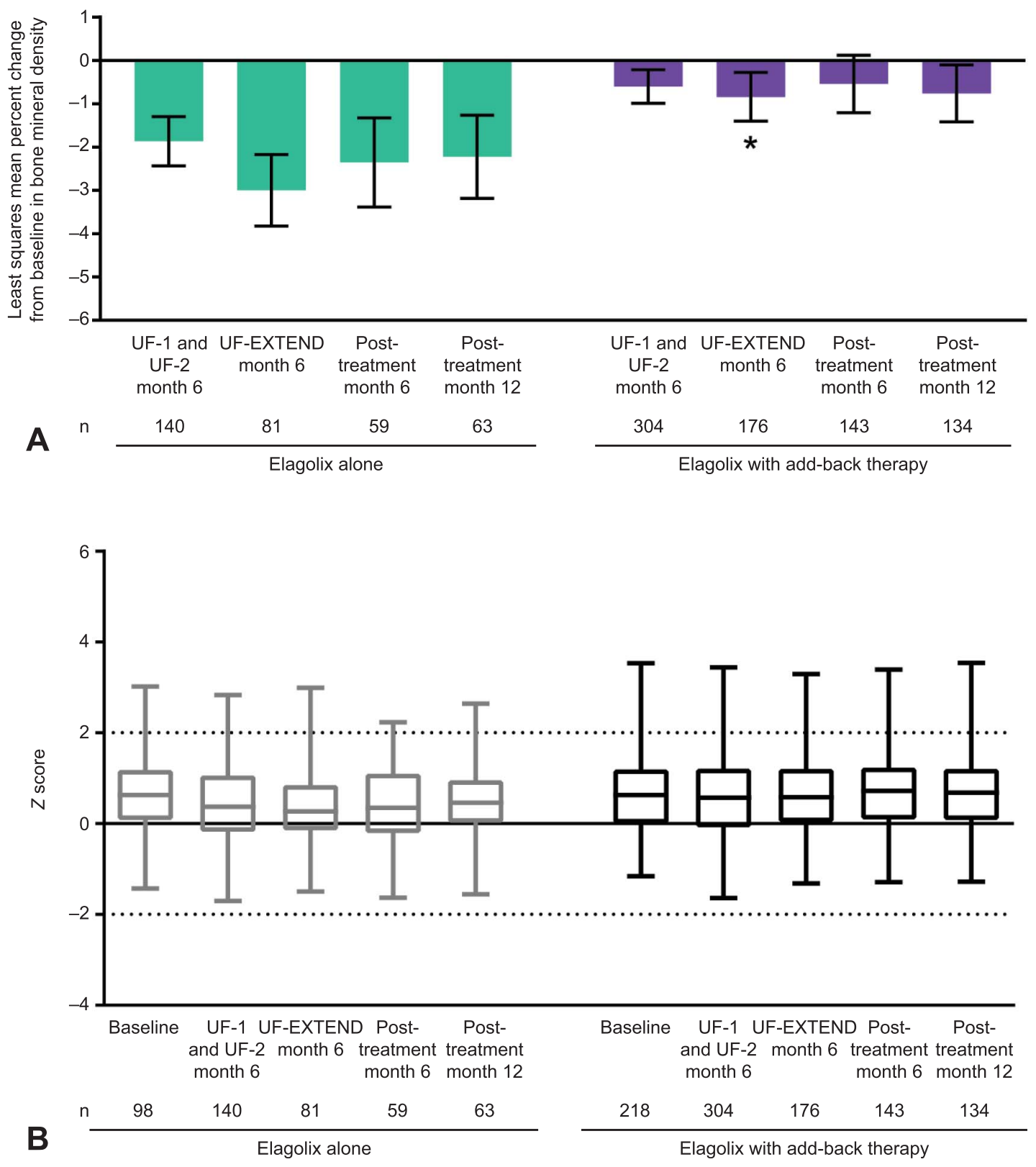

Fig. 5. Femoral neck bone mineral density changes from baseline $(\mathbf{A})$ and bone mineral density Z-scores during treatment and posttreatment periods in women treated with up to 12 months of elagolix (B). Bone mineral density changes are presented as least square mean percent change, with error bars indicating $95 \% \mathrm{Cl}$. Bone mineral density Z-scores are presented as median, quartile 1, quartile 3, and range. Bone mineral density changes and Z-scores for UF-1 and UF-2 exclude participants who switched machine types. Elagolix alone, elagolix $300 \mathrm{mg}$ twice daily throughout UF-1 and UF-2 and UF-EXTEND; elagolix with add-back therapy, elagolix $300 \mathrm{mg}$ twice daily with add-back therapy (estradiol $1 \mathrm{mg}$ and norethindrone $0.5 \mathrm{mg}$ once daily) throughout UF-1 and UF-2 and UF-EXTEND. Baseline was before first dosing in UF-1 and UF-2 studies. ${ }^{*} P \leq .05$ for test of difference between the elagolix with add-back therapy and elagolix-alone groups using an analysis of covariance model with treatment as the main effect and baseline value as a covariate for extension month 6 .

Simon. Twelve Months of Elagolix With Add-Back Therapy. Obstet Gynecol 2020.

UF-EXTEND and, among those with baseline hemoglobin of $10.5 \mathrm{~g} / \mathrm{dL}$ or less, had an increase of more than $2 \mathrm{~g} / \mathrm{dL}$ in hemoglobin after 12 months of treatment $(72.5 \%)$.
Safety findings in Elaris UF-EXTEND were also consistent with those previously reported in the preceding 6 months UF-1 and UF-2 studies; the inclusion of add-back therapy attenuated hypoestrogenic side 
effects associated with the use of elagolix alone, including hot flushes, night sweats, and bone mineral density decreases. There were minimal new cases of either or both hot flushes and night sweats in UF-EXTEND. As expected, elagolix with add-back therapy was associated with numerically lower incidence and reduced severity of either or both hot flushes and night sweats throughout the treatment period based on patient-reported supplemental form data.

The effect of add-back therapy on attenuating bone mineral density changes, as seen with the initial 6 months of treatment in UF-1 and UF-2, were maintained with up to an additional 6 months of treatment in UF-EXTEND, mean percent bone mineral density changes were statistically significantly less with elagolix plus add-back therapy than with elagolix alone. Furthermore, a greater proportion of women in the elagolix with add-back group than in the elagolix-alone group had a numerical increase or no change in bone mineral density. For women who had a numerical decrease in bone mineral density with up to 12 months of elagolix plus add-back therapy, most were in a category of less than $5 \%$ for lumbar spine.

Elagolix with add-back therapy was associated with transient lipid elevations in LDL-C and apolipoprotein $\mathrm{B}$ that were first observed during the first 3 months of treatment in UF-1 and UF-2 and then remained stable; an additional 6 months of treatment in UF-EXTEND did not further increase the lipid levels. The mechanism for these changes is not known, but in most cases increases in concentrations of apoB-containing lipoproteins are explained by a reduction in LDL receptor-mediated clearance often related to decreased receptor expression or function. ${ }^{21,22}$ After study drug discontinuation, lipid levels usually returned to pretreatment levels by month 3 of the posttreatment follow-up period.

There were some methodologic limitations inherent to UF-EXTEND. For example, women were required to complete one of the preceding UF-1 or UF-2 phase 3 studies to enroll in this extension study, resulting in a potential selection bias for women who responded to treatment. The absence of a placebo control in UF-EXTEND also limits the interpretation of the results. Despite these factors, the treatment length is a strength of the extension study, as the safety (including bone mineral density and other hypoestrogenic effects) and efficacy of elagolix with add-back therapy were evaluated for up to 12 months of treatment, and bone mineral density recovery for up to 12 months posttreatment. An additional strength is the inclusion of the elagolix alone reference arm, which was used to characterize the effects of add-back therapy on the hypoestrogenic effects of elagolix. The objective measurement of menstrual blood loss using the alkaline hematin method also adds credibility to the study as other methods, such as the pictorial blood loss assessment chart and bleeding journals, are semiquantitative and subjective. ${ }^{23-25}$

In conclusion, the results of UF-EXTEND demonstrated that up to 12 months of elagolix with addback therapy provided sustained efficacy in reducing menstrual blood loss with no new or unexpected adverse effects compared with results of the preceding 6-month UF-1 and UF-2 studies. Add-back therapy continued to attenuate hypoestrogenic effects associated with the use of elagolix alone, which were consistent across the 12-month treatment course. These data support the evaluation of elagolix with add-back therapy as a potential new, long-term, oral treatment option for women with heavy menstrual bleeding associated with uterine leiomyomas.

\section{REFERENCES}

1. Stewart EA, Nicholson WK, Bradley L, Borah BJ. The burden of uterine fibroids for African-American women: results of a national survey. J Women's Health 2013;22:807-16.

2. Baird DD, Dunson DB, Hill MC, Cousins D, Schectman JM. High cumulative incidence of uterine leiomyoma in black and white women: ultrasound evidence. Am J Obstet Gynecol 2003; 188:100-7.

3. Stewart EA. Uterine fibroids. Lancet 2001;357:293-8.

4. Wegienka G, Baird DD, Hertz-Picciotto I, Harlow SD, Steege JF, Hill MC, et al. Self-reported heavy bleeding associated with uterine leiomyomata. Obstet Gynecol 2003;101:431-7.

5. Napolitano M, Dolce A, Celenza G, Grandone E, Perilli MG, Siragusa S, et al. Iron-dependent erythropoiesis in women with excessive menstrual blood losses and women with normal menses. Ann Hematol 2014;93:557-63.

6. Fuldeore MJ, Soliman AM. Patient-reported prevalence and symptomatic burden of uterine fibroids among women in the United States: findings from a cross-sectional survey analysis. Int J Women's Health 2017;9:403-11.

7. Soliman AM, Margolis MK, Castelli-Haley J, Fuldeore MJ, Owens CD, Coyne KS. Impact of uterine fibroid symptoms on health-related quality of life of US women: evidence from a cross-sectional survey. Curr Med Res Opin 2017;33:1971-8.

8. Ghant MS, Sengoba KS, Recht H, Cameron KA, Lawson AK, Marsh EE. Beyond the physical: a qualitative assessment of the burden of symptomatic uterine fibroids on women's emotional and psychosocial health. J Psychosomatic Res 2015;78:499503.

9. Cardozo ER, Clark AD, Banks NK, Henne MB, Stegmann BJ, Segars JH. The estimated annual cost of uterine leiomyomata in the United States. Am J Obstet Gynecol 2012;206:211.e1-9.

10. Fuldeore M, Yang H, Soliman AM, Winkel C. Healthcare utilization and costs among women diagnosed with uterine fibroids: a longitudinal evaluation for 5 years pre- and postdiagnosis. Curr Med Res Opin 2015;31:1719-31. 
11. Soliman AM, Anand SB, Coyne KS, Castelli-Haley J, Snabes $\mathrm{M}$, Owens CD. Examining the relationship between symptomatic burden and self-reported productivity losses among patients with uterine fibroids in the United States. J Occup Environ Med 2017;59:974-81.

12. Zimmermann A, Bernuit D, Gerlinger C, Schaefers M, Geppert $\mathrm{K}$. Prevalence, symptoms and management of uterine fibroids: an international internet-based survey of 21,746 women. BMC Womens Health 2012;12:6.

13. Stewart EA. Clinical practice. Uterine fibroids. New Engl J Med 2015;372:1646-55.

14. Borah BJ, Yao X, Laughlin-Tommaso SK, Heien HC, Stewart EA. Comparative effectiveness of uterine leiomyoma procedures using a large insurance claims database. Obstet Gynecol 2017;130:1047-56.

15. Donnez J, Dolmans MM. Uterine fibroid management: from the present to the future. Human Reprod Update 2016;22:665-86.

16. Gurusamy KS, Vaughan J, Fraser IS, Best LM, Richards T. Medical therapies for uterine fibroids-a systematic review and network meta-analysis of randomised controlled trials. PLoS One 2016;11:e0149631.

17. Schlaff WD, Ackerman RT, Al-Hendy A, Archer DF, Barnhart KT, Bradley LD, et al. Elagolix for heavy menstrual bleeding in women with uterine fibroids. New Engl J Med 2020;382: 328-40.

18. Magnay JL, Schonicke G, Nevatte TM, O'Brien S, Junge W. Validation of a rapid alkaline hematin technique to measure menstrual blood loss on feminine towels containing superabsorbent polymers. Fertil Steril 2011;96:394-8.

19. Coyne KS, Soliman AM, Margolis MK, Thompson CL, Chwalisz K. Validation of the 4 week recall version of the uterine fibroid symptom and health-related quality of life (UFS-QOL) questionnaire. Curr Med Res Opin 2017;33:193-200.

20. International Society for Clinical Densitometry. Official positions 2015: adult and pediatric. Available at: https://www. iscd.org/official-positions/2015-iscd-official-positions-adult/. Retrieved November 19, 2017.

21. Persson L, Henriksson P, Westerlund E, Hovatta O, Angelin B, Rudling M. Endogenous estrogens lower plasma PCSK9 and LDL cholesterol but not $\mathrm{Lp}(\mathrm{a})$ or bile acid synthesis in women. Arterioscler Thromb Vasc Biol 2012;32:810-4.

22. Ghosh M, Galman C, Rudling M, Angelin B. Influence of physiological changes in endogenous estrogen on circulating PCSK9 and LDL cholesterol. J Lipid Res 2015;56:463-9.
23. Higham JM, O'Brien PM, Shaw RW. Assessment of menstrual blood loss using a pictorial chart. Br J Obstet Gynaecol 1990; 97:734-9.

24. Mansfield PK, Voda A, Allison G. Validating a pencil-andpaper measure of perimenopausal menstrual blood loss. Womens Health Issues 2004;14:242-7.

25. Reid PC, Coker A, Coltart R. Assessment of menstrual blood loss using a pictorial chart: a validation study. BJOG 2000;107: $320-2$.

\section{Authors' Data Sharing Statement}

AbbVie Inc. is committed to responsible data sharing regarding the clinical trials we sponsor. This includes access to anonymized, individual and trial-level data (analysis data sets), as well as other information (e.g., protocols and Clinical Study Reports), as long as the trials are not part of an ongoing or planned regulatory submission. This includes requests for clinical trial data for unlicensed products and indications.

This clinical trial data can be requested by any qualified researchers who engage in rigorous, independent scientific research, and will be provided following review and approval of a research proposal and Statistical Analysis Plan (SAP) and execution of a Data Sharing Agreement (DSA). Data requests can be submitted at any time and the data will be accessible for 12 months, with possible extensions considered. For more information on the process, or to submit a request, visit the following link: https://www.abbvie.com/our-science/clinicaltrials/clinical-trials-data-and-information-sharing/dataand-information-sharing-with-qualified-researchers.html.

\section{PEER REVIEW HISTORY}

Received December 2, 2019. Received in revised form February 13, 2020. Accepted February 20, 2020. Peer reviews are available at http://links.lww.com/AOG/B844. 